\section{De Tolkien al LitRPG: MMORPGS y transmediación}

Martín M. Vizzotti ${ }^{(1)}$

Resumen: En este artículo recorremos un derrotero transmediático que tiene como resultado el surgimiento de un nuevo género literario, el LitRPG (Literary RPG). Este proceso comienza en la década del 70 cuando se interactivizan ciertas literaturas y autores eminentes como Tolkien, Lovecraft, Moorcock, Howard et al. y aparecen los primeros RPG (Role Playing Games), luego, estos son transmediados a los nuevos soportes cibernéticos para dar origen a los CRPG (Computer RPG), MUDs (Multi User Domain/ Dungeon) y finalmente los MMORPG (Massive Multiplayer Online RPG), cuyas lógicas y características discursivas irrumpen en el terreno literario a través de las obras de LitRPG y generan un regreso a la literatura a partir de un proceso de desinteractivación.

Además de analizar el proceso de transmediación, se analizan también las características principales de este nuevo género, adentrándonos en la obra de cuatro autores considerados pioneros: D. Rus, G. Akella, V. Mahanenko y Yu Wo, así como también en ciertas características prefiguradas en ciertos precursores del género (en términos de "Kafka y sus precursores", de J. L. Borges).

Palabras clave: LitRPG - D. Rus - G. Akella - V. Mahanenko - Yu Wo - Transmediación - RPG - MMORPG

[Resúmenes en inglés y portugués en las páginas 157-158]

(1) Profesor, Licenciado y Doctor en Letras de la Facultad de Humanidades y Ciencias de la Educación de la Universidad Nacional de La Plata. Es docente en el Área de Latín desde el año 2000 y Profesor Adjunto ordinario en el área desde 2015. Ha dictado numerosos seminarios de grado y posgrado y publicado artículos en revistas Nacionales e Internacionales. 


\section{Transmediación}

La transmediación ha existido prácticamente desde el inicio de las representaciones artísticas, siendo una de las primeras manifestaciones de este fenómeno el pasaje de lo oral a lo pictórico: comenzando con las pinturas rupestres sobre los relatos de cacería narrados alrededor del fuego, pasando por las pinturas de hazañas y eventos mitológicos en ánforas y vasos de la antigüedad hasta llegar a las complejas series de vitrales que representaban los hechos bíblicos en las iglesias (Konzak, 2018, p. 135). Según Lars Konzak, para poder analizar los procesos y efectos producidos por la transmediación, es fundamental definir qué se entiende por el fenómeno de mediación: este concepto puede hacer referencia o bien a las realidades creadas por los medios implicados, o bien puede ser considerado en relación a la materialidad de estos medios. Para nuestro trabajo nos concentraremos en la segunda acepción del término para analizar cómo resultan mediados ciertos mundos posibles o subcreaciones (Tolkien, 1975, pp. 40-41, 50-52 y 55) a lo largo de un derrotero que implica varias transformaciones mediáticas que los somete a un proceso dinámico de alteraciones y adaptaciones a medida que son transmediados de un soporte a otro.

Existen cinco formas de adaptación transmediáticas: 1) descripción, 2) visualización, 3) auralización, 4) interactivación y 5) desinteractivación (Konzak, 2018, pp. 134-135). La descripción se da cuando una obra visual es trasladada a un formato escrito, por ejemplo ${ }^{1}$, la novelización para niños de los episodios IV, V y VI de Star Wars por Alexandra Bracken, Adam Gidwitz o Tom Angleberger; o la novelización de los episodios de Star Trek por parte de James Blish a fines de los 60 y principios de los $70^{2}$.

La visualización ocurre cuando una obra de arte escrita se vuelca a un formato visual, estático o dinámico, como en el caso de la trilogía de El Señor de los anillos, de Peter Jackson $(2001,2002,2003)$. Auralización es cuando un texto es transformado a un formato de representación sonoro, como la eminente y performativa emisión radial de Orson Welles de La Guerra de los Mundos, el 30 de Octubre de 1938 a través de la CBD Radio. Por otro lado, interactivación y desinteractivación son procesos opuestos, la primera implica la transformación de una obra lineal o no-interactiva a un formato que permita diversos grados de interacción, por ejemplo cualquier RPG (Role Playing Game) o CRPG (Computer Role Playing Game) basado o inspirado en algunos de los muchos mundos secundarios tales como la Tierra Media de Tolkien: desde la primitiva, aunque fundacional, text-adventure de Beam Software The Hobbit (1982), hasta el visualmente impresionante Middle-earth: Shadow of War (2017), de Monolith Productions y publicado por Warner Bros. La desinteractivación implica, obviamente, que una manifestación artística interactiva es transformada en otra obra no interactiva, por ejemplo la novelización del excelente CRPG Planescape: Torment (1999), de Black Isle Studios, por parte de Ray y Valerie Vallese editado por Wizard of the Coast, o la más cercana al original de Rhyss Hess, con textos originales del juego pertenecientes a sus desarrolladores, Chris Avellone y Colin McComb ${ }^{3}$. 


\section{El origen y la evolución de los RPG y los CRPG}

Los RPG propiamente dichos nacen a principios de la década del 70 combinando las reglas básicas de los juegos de guerra y estrategia y ciertos aspectos y técnicas de los juegos de rol utilizados en actuación e incluso en el psicoanálisis. El juego de simulación de guerra más eminente es, por supuesto, el ajedrez, cuyas reglas permanecen incólumes desde hace más de 1500 años, sin embargo, entre los siglos XVII y XIX, éste fue modificado para incluir las circunstancias lábiles de todo combate y dar cuenta de los azares de la guerra ${ }^{4}$. De hecho, muchas simulaciones de batallas con estas características han sido documentadas a lo largo de la historia, por ejemplo, son famosos los episodios ocurridos el 1 y el 25 de Mayo de 1942 a bordo del acorazado Yamato, cuando los altos mandos japoneses desestimaron varias simulaciones de la futura batalla de Midway que predijeron, con asombrosa exactitud, los desastrosos resultados de la misma (Parshall, \& Tully, 2007, pp. 61-64 y 67-69). En 1971 Gary Gygax y Jeff Perren desarrollan Chainmail, un juego de simulación de combates medievales, el cual agregaba la novedad de permitir la presencia de elementos fantásticos como magos y dragones. Poco a poco, el énfasis de los juegos fue variando y las unidades de combate en miniatura fueron reemplazadas por personajes individuales, cada uno con sus motivaciones y personalidades particulares, tal como sucede en Blackmoor, de Dave Arneson. Finalmente, en 1974, Gygax y Arneson crean Dungeons and Dragons (D\&D) (Barton, 2008, pp. 16-20; Peterson, 2012, pp. 41-46 y 64-71): el juego fue un furor entre los amantes de la literatura fantástica y de los juegos de guerra y a partir de allí surgen infinitas versiones y adaptaciones, ya sean éstas de los diferentes settings - medievales, fantásticos, góticos, cyberpunks, steampunks, espaciales y/o sus combinaciones posiblessino también de la mecánica del juego en general, dependiendo del énfasis y los intereses de cada grupo de jugadores (Tresca, 2011, pp. 59-62.; Barton, 2008, pp. 21-23.).

En lo que respecta a nuestro trabajo, la influencia de Tolkien en la creación de D\&D es pervasiva y evidente, basta ver las diferentes razas ofrecidas por el juego: elfos, humanos, enanos y medianos (Tresca, 2011, pp. 23-25; Peterson, 2012, pp. 116-120; Barton, 2008, pp. 17-19). Por supuesto que las influencias no están circunscriptas sólo a Tolkien, pueden encontrarse en el juego la influencia de numerosos autores, por ejemplo, Moorcock, Howard, Lovecraft y muchos más (El propio Gary Gygax nos ofrece sus Inspirational and educational readings en Gygax, 1979, p. 224) .

Los RPG, en definitiva, son un claro ejemplo de la interactivación de ciertos campos específicos de la literatura y ciertos autores particulares, donde las posibilidades lineales de las narraciones son ampliadas ad infinitum y las líneas argumentales y los ámbitos explicitados y/o sugeridos en las obras son explorados, renovados y reformulados. El paso de los RPG a los CRPG implica, por su parte, una conjunción de transmedialidades: en primer lugar, existe una disminución en el grado de interactividad, desde las infinitas posibilidades de los RPG a las más limitadas posibilidades de los CRPG (debido a los requerimientos de este nuevo soporte), pero, sin embargo, se agregan las potencialidades ofrecidas por la visualización y la auralización. 
La historia de los CRPG es también, por supuesto, intrincada y compleja, por lo que nos enfocaremos sólo en ciertos aspectos que consideramos relevantes para este trabajo ${ }^{6}$. Existen variadas taxonomías en relación a los juegos de PC relacionados a la rama de los RPG, de acuerdo a los diferentes énfasis de cada categoría. Matt Barton, al clasificarlos, hace las siguientes distinciones: CRPG vs. juegos de aventuras; CRPG vs. juegos de estrategia y, más relevante para nuestro trabajo, CRPG vs. MUDs y MMORPG (Barton, 2008, pp. 5-10.). En la década del 70, con las rudimentarias y limitadísimas herramientas de la época, se crearon los primeros CRPG que intentaban recrear o, en la terminología actual, transmediar la experiencia interactiva de los RPG al soporte de las nuevas PC (Barton, 2008, pp. 29 y ss.). La mayoría de estos programas pioneros hacen referencia en sus títulos al mundo secundario literario que inspiró, como ya vimos, los RPG y/o hacen un claro homenaje a su creador, J. R. R. Tolkien: Akalabeth. World of Doom (1978), Moria (1978) y Orthanc (1978) fueron desarrollados para la plataforma PLATO (Programmed Logic Automated Teaching Operations), que nació como un programa de asistencia educativa a distancia en la Universidad de Illinois en la década del 60 (Barton, 2007 y 2008, pp. 32-33). En estos años aparecen también los primeros MUDs (Multi User Domain, aunque debido a su uso eminentemente relacionado a los RPG, el acrónimo pasó a significar Multi User Dungeons), de cuya evolución surgen los primeros MMORPG (Massive Multiplayer Online RPG). Los MUDs, grosso modo, ofrecen el ámbito cibernético para desarrollar sesiones de rol o diferentes aventuras interactivas, usualmente a través de textos y quizás algunos gráficos. Según M. Barton, los MUDs suelen tender hacia la interacción social (role-playing), mientras que los MMORPG se centran en los aspectos relacionados al roll-playing, donde el énfasis y la atención están basados en las tiradas de dados (hack and slash), el looting y el leveling (Barton: 2008, 42). En definitiva, en los MMORPG, los jugadores interactúan dentro de un mundo virtual de acuerdo a ciertas reglas y ciertas lógicas propias de esos mundos secundarios, de los cuales, como hemos visto, si bien estos mundos pueden tener, al igual que los RPG y los CRPG, ambientaciones muy diferentes, los más conspicuos son los ambientados en mundos fantásticos.

\section{LitRPG: precursores, particularidades y características}

It started in mud, as many things do

(Williams, 1998)

Tzvetan Todorov, en su Introducción a la Literatura Fantástica, se pregunta si “[...] tenemos derecho a discutir un género sin haber estudiado (o al menos leído) todas las obras que lo constituyen", aunque cabe destacar que cuando habla de género, distingue sólo entre épico, poético, trágico y cómico (2006, p. 1-3). Darko Suvin, por su parte, en su fundacional Metamorphoses of Science Fiction, considera el género como un repertorio de ciertas funciones, convenciones y procedimientos específicos verificables empíricamente (Suvin, 1977, pp.10-11 y 63-65.) y, finalmente, coincide con T. Todorov en que es posible y válido analizar un género literario a partir de sus obras más representativas, aunque, dentro de su ideología específica, él habla de ciertas masterpieces: 
Finalmente, será posible esbozar las premisas básicas de una crítica, una historia y de una teoría significativa de este género [...] La primera podría ser que un género debe ser evaluado procediendo desde su cima hacia abajo, aplicando los estándares obtenidos del análisis de sus obras maestras. (Suvin: 1977, 36.) ${ }^{7}$

Más allá de la perspectiva axiológica particular de Suvin, cuya discusión excede los límites de este trabajo, el criterio resulta válido. Para nuestro trabajo analizaremos cuatro autores fundacionales del género: Yu Wo, D. Rus, V. Mahanenko y G. Akella, también haremos referencia a ciertas obras "precursoras" del género (tal como está planteado en "Kafka y sus precursores", de J. L. Borges), como Otherland: City of Golden Shadows (1998), de Tad Williams, Epic (2004), de Connor Konstik o Ready Player One (2011), de Ernest Cline, visualizada muy libremente por Steven Spielberg en 2018, donde ciertos procedimientos, ideas y extrapolaciones fundamentales a las obras estudiadas están presentes o aparecen de manera seminal. Consideramos relevante detenernos brevemente en estas cuestiones ya que, como todo nicho literario y económico, el éxito del LitRPG ha generado una explosión de oferta oficial y no-oficial (fan fiction) tanto en tiendas mainstream (Amazon, Barnes \& Noble, etc.) como en los blogs y sitios de fans. Según V. Mahanenko, la etiqueta LitRPG para el género fue acuñada en Rusia en el año 2013 cuando él, junto al escritor A. Bobl y a Dmitri Malkin, editor de Sci-Fi para la compañía Eksmo (Эксмо, una de las editoriales más importantes de Rusia), durante una sesión de brainstorming. De allí surge la extrañeza gramatical del término LitRPG (Literary RPG) frente al quizás más esperable, aunque anglocéntrico, RPGLit (RPG literature) (Konstik, 2018). Básicamente, para que un relato o narración pueda sea considerada como parte del género LitRPG, la acción debe desarrollarse, al menos en gran parte, dentro de un entorno virtual y bajo condiciones de full immersion: este es el componente de ciencia ficción del juego, la extrapolación de una tecnología existente a un parámetro superior aún no alcanzado. Sin embargo, el LitRPG propiamente dicho debe poseer otro elemento que consideramos constitutivo y distintivo: la trama debe desarrollarse en el entorno de un MMORPG, bajo la égida de sus leyes intrínsecas, sus lógicas y sus convenciones.

\section{Los precursores}

Tad Williams, con su serie Otherland (1996-2004), Connor Konstik con Epic (2004) y Ernest Cline con Ready Player One (2011), son considerados los "precursores" del género. En Otherland: City of Golden Shadows (1996) y en Epic (2004) se despliegan procedimientos y tópicos que estarán presentes en las obras de LitRPG -la acción transcurre dentro de un entorno virtual, con full immersion lograda a través de ciertos avances tecnológicos específicos. No aparecen, sin embargo, ciertos elementos que constituyen, creemos, el núcleo central de este género: el ámbito específico de un MMORPG, su terminología ni sus lógicas particulares. Ready Player One está ya más cerca de esto: el entorno virtual donde se desarrolla la acción es un MMORPG y los jugadores, o gunters, enfrentan diferentes pruebas inspiradas en clásicos juegos y películas de las últimas décadas del siglo XX; sí están presentes, aunque no en un primerísimo plano ni de manera pervasiva (como sucede 
en la obra de Rus, Mahanenko, Akella y Wo), ciertos detalle inherentes a la jugabilidad y a la lógica interna de los MMORPG: las estadísticas del personaje, el método de creación, los puntos de experiencia y el leveling, las interfaces varias, etc. Lo cual nos lleva al LitRPG propiamente dicho.

\title{
El LitRPG
}

Así como existe una ciencia ficción "dura", donde muchas veces los elementos extraliterarios que pretenden ofrecer el novum y el "extrañamiento cognitivo" que celebra Suvin (1977, pp. 4-5; 11-15 y 63-67) resultan más relevantes que las cuestiones literarias propiamente dichas, por ejemplo, Nightfall (1941) o The Dying Night (1956) de I. Asimov. En el caso del LitRPG resulta central la aparición pervasiva de las convenciones, los términos y las lógicas propias de un MMORPG, así como las irrupciones de ciertos mensajes específicos dentro de la interfaz del protagonista:

\author{
¡Felicitaciones! Has aprendido un hechizo: La mano del Muerto. \\ Tiempo de lanzamiento: $1.5 \mathrm{seg}$. \\ Gasto de Maná: 19 \\ Congela el blanco por 2 seg. \\ (Rus, 2014, cap. 5, párr. 36)
}

La terminología es clara y transparente, casi económica para el iniciado y absolutamente oscura para el lego. Es esta intrusión de un canal y una terminología específica, completamente ajena al verosímil fantástico, donde yace, creemos, la originalidad primordial del género: un lenguaje técnico, casi no-literario y específico de un campo semántico muy acotado que irrumpe en el campo literario y configura sus propias lógicas de enunciación y es a su vez regido sólo por ellas:

Se lanzó contra el mob, acuchillándolo cada vez que estaba a su alcance. Pero no podía retirarse lo suficientemente rápido ya que había excitado demasiado el aggro del mob. Ignorando a su oponente, el monstruo vino por mí.

¡Fuiste golpeado por el Gnoll mensajero! Daño recibido 16 puntos. Vida 44/60

¡Fuiste golpeado por el Gnoll mensajero! Daño recibido 12 puntos. Vida 32/60

Eso dolió (Rus, 2014, cap. 4, párrs. 68- 71)

Esta última función genera otra de las características principales del verosímil propio del LitRPG: las lógicas que rigen los combates del LitRPG nada tiene que ver con la representación habitual en los distintos relatos de fantasía. G. Akella va más allá de lo necesario para explicar esto en su novela Patch 17: 
La mecánica de combate de los RPG, que involucran grupos y redadas a calabozos y otras misiones, casi no ha cambiado en los últimos treinta y cuatro años. Cada expedición consta de tres roles: tanques - jugadores cuyo rol es mantener la atención de los jefes o mobs, atrayendo más aggro que el resto de los jugadores y así protegiendo al resto del grupo o de la expedición de recibir daño. Sanadores- personajes que restauran o mantienen la salud del grupo o la expedición durante el combate. Y finalmente, dps (daño por segundo) - personajes cuya función central es infligir daño a los jugadores enemigos, NPC (mobs) y jefes [...] Si por alguna razón el tanque pierde el aggro, permitiendo al jefe alejarse y atacar a los débiles sanadores o dps, en casi todos los casos el resultado es una limpieza, es decir, la muerte de la expedición completa. (Akella, 2016, cap. 4, párrs. 94-98 y 100)

Comparemos esta representación de los combates con dos de los autores más representativos de la literatura fantástica, R. E. Howard y J. R. R. Tolkien:

Con su espalda contra la pared enfrentó por un instante el círculo que se cerraba sobre él, entonces saltó en medio de ellos. No era un luchador defensivo, incluso ante posibilidades abrumadoramente adversas siempre llevaba la lucha hacia el enemigo. [...] El Cimerio avanzó, un movimiento de velocidad deslumbrante. Era como un tigre entre mandriles cuando saltaba, esquivaba y giraba, ofreciendo un blanco siempre en movimiento, mientras que su hacha tejía una rueda de muerte a su alrededor. (Howard, 2015, "The Phoenix on the Sword”, cap. 5, párrs. 18-19.)

Boromir se adelantó y cortó el brazo con todas sus fuerzas; pero su espada resonó, se desvió y cayó de sus manos sacudidas. La hoja se melló. De repente, para su sorpresa, Frodo sintió una ira ardiente encenderse en su corazón. “¡Por la Comarca!" gritó, y saltando junto a Boromir, se inclinó y apuñaló con Aguijón el horrible pie. Hubo un bramido, y el pie se retiró de repente, casi arrancando a Aguijón de sus manos. Negras gotas cayeron desde el filo y humearon en el suelo. Boromir se arrojó contra la puerta y la cerró nuevamente. “¡Una por la Comarca!” gritó Aragorn. “La mordida del hobbit es profunda! ¡Tienes una buena hoja, Frodo hijo de Drogo!” (Tolkien, 2010, pp. 324-325)

Las estrategias y los métodos de representación son, claramente, diferentes. La descripción de Conan luchando en su habitación contra los conjurados o el combate en la cámara de Mazarbul, junto a la tumba de Balin, en las minas de Moria presentan diferentes estrategias y procedimientos de representación. Veamos un ejemplo quizás extremo, que es el primer combate de Laith, personaje principal de Alterworld ${ }^{8}$ : 
Un conejo adulto. Nivel 3.

Lo que sea. Me está atacando. Vergonzoso, en verdad: ser matado en tu primera lucha -por un conejo. Conejo o no conejo, me apresuré a mover el blando del gran bastardo y lancé un DoT retardador. Resistencia. El blanco ni le preocupa el hechizo. Un conejo con resistencia mágica -¿qué tipo de mundo era éste? Otro intento, y logré retrasarlo un poco.

¡Fuiste mordido! Daño recibido: 6 puntos. Fuente: los dientes del conejo joven. Vida 54/60.

¡El conejo joven ha errado! ¡Intenta golpear, pero falló! (Rus, 2014, cap. 4, párrs.3-7)

Otra de las características centrales de las obras que hemos seleccionado es la relevancia central del proceso de creación del avatar del jugador o personaje principal, que oficia como procedimiento literario de singularización y originalidad y que da pie a otras operaciones y procedimientos narrativos dentro de la trama. Usualmente, en los relatos de fantasía, el héroe posee cierto atributo que, a la corta o a la larga, lo singularizan y, en definitiva, lo convierten en el héroe del relato (como, por ejemplo, las relatos englobados por el periplo del héroe (Campbell, 2008) o los motivos populares como los de Ugly Duckling, Hidden Monarch o Pariah Elite (presentes, por ejemplo, en la construcción del personaje de Aragorn), por nombrar algunos de los más conspicuos (Clute \& Grant, 1997, pp. 972 973; 446; 745-746.). En el caso de Otherland, la singularización no se produce dentro del entorno virtual o debido a alguna característica de los avatares, sino a los contextos personales de los protagonista, especialmente la particular perspectiva bosquimana de !Xabbu. En Ready Player One tampoco está explicitado el proceso de creación de los avatares de los personajes y, aunque sí vemos el proceso de leveling de Parzival, sin embargo, éste se adecúa más a una lógica de los relatos de rags to riches, en los cuales un personaje astuto e inteligente logra ascender hasta lo más alto de la pirámide social, en este caso, tanto en el mundo "real" como en el virtual (rags to legend).

Es habitual en el LitRPG resaltar la especificidad y singularidad del proceso de creación de los avatares. Este procedimiento aporta tensión y profundidad al personaje y al proceso mismo, pues de lo contrario los avatares serían "descartables" y, por lo tanto, poco relevantes o interesantes. Veamos cómo los autores refuerzan esta cuestión en el propio texto:

Bien, puedes ahora comenzar a crear a tu personaje. Antes de proceder, debo recordarte que sólo tendrás una oportunidad para crear tu personaje. Una vez creado, tu raza, nombre y apariencia no pueden ser cambiados. (Yu Wo, 2004, p. 16). ¡Felicitaciones! Recibes un $+1 \%$ de bono por clase a Intelecto y +1 a Espíritu en cada nivel. Elije tus características iniciales. Tienes 25 puntos. Úsalos sabiamente. Una vez que el personaje ha sido creado, no pueden realizarse más cambios. La descripción de los cinco atributos flotó ante mis ojos. (Rus,2014, cap. 3, párrs. 14-15) 
Además, dentro de la relevancia narrativa y la singularidad del proceso de creación, aparece usualmente, como ya mencionamos, cierto rasgo único que elevará al personaje y destacará al avatar de éste dentro de la población general del dominio digital. Feng Lan, protagonista principal de la saga de Yu Wo, resulta ser la primera jugadora en conectarse a Second Life, un MMORPG con un 99\% de realismo, y, por esta razón, le es concedido un deseo (Yu Wo, 2004, pp. 17-18).

D. Rus, por su parte, singulariza a su personaje, y al avatar de éste, con la particular elección de su clase:

Introduje la información de la tarjeta de crédito en el registro, ignorando la interminable lista de palabras de la descripción, fui derecho al menú de creación del personaje. Elegir personaje. [...] Elija su clase. “Brujo.” Información: Los Brujos son los adoradores secretos del Señor Caído y se ven atraídos hacia las Fuerzas oscuras. Las razas de la luz tienden a rechazarlos. Ciertos NPC pueden rehusarse a interactuar contigo. Muchos comerciantes pueden inflar sus precios al tratar contigo. Al alcanzar el nivel 10, un Brujo deberá decidir su especialización. Se le requerirá elegir entre Nigromante o Caballero de la Muerte. Ambos son despreciados por los poderes de la Luz. Muchas misiones y locaciones estarán vedadas. Si aún desea jugar con la clase en cuestión, sugerimos que elija una raza Oscura o neutral. "Ni loco!" grité a la interfaz, "Necro es mi toon favorito desde que nací! No quiero ser el enésimo arquero elfo. Me importa un bledo tus estándares políticamente correctos. "Aconsejamos, sugerimos, quizás sea mejor...” Sí, seguro. Voy a cagar tu esquema. Seré el primer Alto Elfo Oscuro entre tu lindos y bonitos Paladines. ¡Confirmar!” (Rus, 2014, cap. 3, párr. 2 y 10-15.)

En los casos de Survival Quest y Patch 17, los personajes se ven forzados, uno por decisión judicial (Mahan), el otro a punta de pistola (Krian), a encarnar personajes específicos: Krian es lanzado a un territorio desconocido no habilitado aún para la masa de jugadores y bajo la especie de un demonio:

Bienvenido al Reino de Arkon, Krian. Ahí estaba la familiar oscuridad de la pantalla de log-in. ERROR 757@ 4\#! 278\% \$ El menú de selección de raza apareció nuevamente. Demonio. Cada representante de esta raza lleva una gota de sangre del Señor del Inframundo dentro de sí.... ERROR $757 \$ \% * \&$ ^ ... llevando armadura pesada, son algunos de los mejores guerreros del Reino de Akron. Obtienes un $75 \%(\max )$ de bonificación en resistencia a la magia mental, la habilidad de usar armaduras pesadas, $y$ un $+2 \%$ a Armadura cuando la usas. ERROR 757\%\# \$@*)\& ¿Demonio? ¡Qué demonios! Se suponía que jugaría con un humano. ¡Los demonios no habían sido incluidos en el Parche aún! (Akella, 2016, Intr. párrs. 1-9) 
Mahan, por su parte, es encarcelado en una mina de trabajos forzados virtual para pagar su deuda a la sociedad (elemento prefigurado en Ready Player One, cuando Parzival se hace encarcelar en las instalaciones de trabajo forzado de IOI) y obligado a aceptar una clase y unas habilidades poco prometedoras:

Ningún bastardo había escrito aún en ningún foro que un prisionero podía recibir una clase con hechizos. ¡Joder! Ni siquiera sé cómo lanzar hechizos y aprenderlo solo va a ser muy difícil.

Hice aparecer la ventana con la descripción del personaje ante mis ojos:

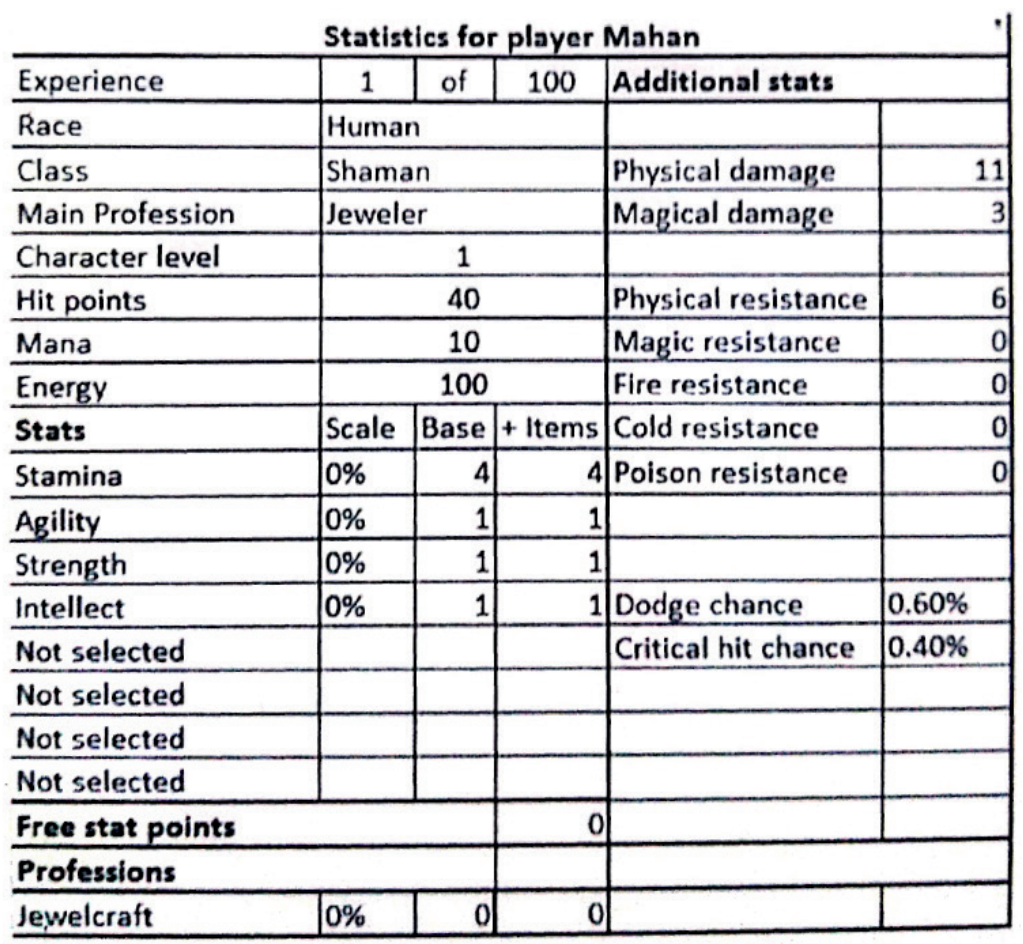

(Mahanenko, 2015, cap. 2, Párrs. 5-6)

Nuevamente queda en un primer plano la irrupción de la interfaz del jugador dentro de la obra y como procedimiento que permite transmitir gran cantidad de información de manera condensada y económica sin alterar en absoluto los parámetros de este nuevo verosímil. Este procedimiento de singularización de los avatares a través de ciertos eventos y circunstancias particulares ofrece opera literariamente y resulta central para el desarrollo y la 
originalidad de la trama, ya que permite, nuevamente, la irrupción en la literatura de un procedimiento propio de la lógica de los CRPG y, particularmente, de los MMORPG, las diferentes misiones, o quests, que los personajes reciben y que, al ser cumplidas, permiten que el avatar progrese y suba de nivel (leveling). Sin embargo, las características particulares de los avatares centrales abren la puerta a otro procedimiento de singularización que resultará aún más importante para la trama de estos relatos: las muy deseadas y buscadas misiones secretas u ocultas, hidden quests. Akella, nuevamente, va un poco más allá de lo aconsejable en su explicación:

Las misiones ocultas son muy apreciadas por todos los jugadores. Conseguir una sólo es posible estando en el lugar correcto en el momento correcto, y luego cumplir un montón de condiciones para desarrollarlas. (Akella, 2016, cap. 2, párr. 150)

Las particulares circunstancias que rodean a su avatar, Krian, le permiten a su vez acceder a estas raras y exclusivas posibilidades:

El hecho de que hubiera recibido una misión de una NPC de nivel 500 sólo podía ser explicado gracias a una anomalía del Patch RP-17, que mencionaba algo sobre un bache en la inteligencia de los NPC.... Quienquiera que estaba monitoreando los territorios demoníacos debe estar dándome estas misiones porque estoy sólo yo aquí. No es que me queje, por cierto. (Akella, 2016, cap. 2, párrs. 316 y 422.)

En Alterworld, gracias a la particular elección de clase del personaje, accede a misiones que no están disponibles para el grueso de los jugadores:

¡Alerta de misión cumplida! Has completado una misión secreta:

Hermandad Oscura.

Recompensa: 1 oro.

Tu relación con Gunnar ha mejorado.

¡Felicitaciones! Has recibido un Logro: El primero en la ciudad.

Te has convertido en la primera persona en esta ciudad en completar la misión:

Hermandad Oscura.

Recompensa: +100 a Fama.

[...]

¡Alerta de nueva misión! Hermandad Oscura II.

Tipo de misión: Secreta, infrecuente.

Encuentra a los seguidores del Señor Caído en la ciudad y en la Tierra de Luz. Cada nuevo adorador doblará la recompensa.

¿Quieres aceptar la misión? (Rus, 2014, cap. 6, párrs. 21-26 y 29-32.)

La concatenación de quests permite al personaje principal singularizarse y evolucionar tanto en el marco lúdico como en el literario, donde nuevamente irrumpen las lógicas discursivas y argumentales de los MMORPG. Dado el gran éxito obtenido en el terreno comercial, el LitRPG es un género prolífico, no sólo los autores más reconocidos producen 
a un ritmo admirable sagas extensísimas (Play to Live se extiende siete volúmenes, como también lo hace Way of the Shaman, y Yu Wo, por su parte, ha escrito nueve volúmenes), hay que sumarle el ejército de nuevos autores que se suman al boom, literario y editorial, y los ya mencionados fan-fictions que se publican en portales de los fanáticos. Sin embargo, hemos encontrado ciertos topoi comunes en las obras analizadas que, sin pretensión alguna de ser exhaustivos, hemos resumido en el siguiente cuadro:

\begin{tabular}{|c|c|c|}
\hline \multicolumn{2}{|l|}{ Precursores } & \multirow{2}{*}{$\begin{array}{c}\text { LitRPG } \\
\text { AlterWorld/ Patch } 17 \\
\text { 1/2 Prince/ Survival Quest }\end{array}$} \\
\hline Otherland/ Epic & Ready Player One & \\
\hline $\begin{array}{l}\text { Entorno virtual } \\
\text { Full Immersion }\end{array}$ & $\begin{array}{l}\text { Entorno virtual } \\
\text { Full Immersion }\end{array}$ & $\begin{array}{l}\text { Entorno virtual } \\
\text { Full Immersion }\end{array}$ \\
\hline$X$ & $\begin{array}{l}\text { La acción se desarrolla } \\
\text { mayormente en un } \\
\text { MMORPG }\end{array}$ & $\begin{array}{l}\text { La acción se desarrolla } \\
\text { casi en su totalidad en un } \\
\text { MMORPG }\end{array}$ \\
\hline$x$ & $\begin{array}{l}\text { Aparecen referencias } \\
\text { a la interfaz del jugador }\end{array}$ & La interfaz irrumpe en la narración. \\
\hline $\mathrm{x}$ & $x$ & $\begin{array}{l}\text { La creación del avatar } \\
\text { aparece como elemento central } \\
\text { para el desarrollo } \\
\text { de la acción y como elemento } \\
\text { de singularización } \\
\text { del personaje. }\end{array}$ \\
\hline
\end{tabular}

Existen también otras características que no resultarían distintivas del género per se, pero que también pueden encontrarse en la mayoría de las obras. En primer lugar, la aparición de eventos relevantes del mundo "real" suele introducirse en el relato a través de un procedimiento bastante habitual, la referencia a memos empresariales, informes y/o noticias oficiales: 
Una vez que había saciado mi apetito primario, me senté con la taza de café siempre caliente y abrí las noticias. Primero revisé los titulares del mundo real para lo que quería saber [...] El trágico accidente de la semana pasada cuando la facción militante del grupo radical Femen hizo estallar ambos centros de datos del mundo virtual de Padisha causó que nuestros lectores se conmiseraran profundamente con las familias de más de mil jugadores permanentes que habitaban el mundo de Padisha junto con los más de ochocientos mil jugadores regulares. Ayer, la base de datos de respaldo fue finalmente instalada en los nuevos servidores del juego. Para alivio de todos, los individuos digitales demostraron estar sanos y salvos e incluso habían alcanzado variados progresos en el juego. Expresaron su sorpresa por la ausencia de los jugadores regulares durante toda la semana. El gobierno aún no se ha explayado sobre este evento extraordinario. (Rus, 2014, cap. 18, párrs. 8 y 14.)

Elegimos este fragmento en particular porque introduce, en su economía informativa, otro de los elementos característicos del género: la inminente sospecha de que el mundo "virtual" se está volviendo independiente de sus soportes en el mundo "real", con la consecuente posibilidad de la existencia independiente de las AI y de los NPC. El final de Alterworld: Play to Live es un ejemplo diáfano de esto, así como la creciente sospecha de los personajes de Patch 17 sobre la existencia independiente e individual de los NPC.

\section{Conclusiones}

A lo largo de nuestra argumentación hemos visto cómo ciertos elementos presentes en la obra de destacados autores fantásticos, especialmente Tolkien, han pasado por un complejo y dinámico proceso de transmediación: la interactivación de los mundos secundarios o subcreaciones y su hibridación con ciertos juegos de estrategia y de simulación de combates dieron como resultado, a mediados de la década del 70, la aparición de los RPG, aportando así infinitas posibilidades de expansión a las líneas y concepciones presentes en estas obras literarias lineales. Luego, a medida que avanzaban las posibilidades técnicas, los RPG eran transmediados a los diferentes soportes cibernéticos, apareciendo entonces los CRPG, los MUDs y, finalmente, los MMORPG, adquiriendo cada una, a lo largo de su evolución, sus propias lógicas internas y sus propias reglas intrínsecas de interacción. A partir de estas lógicas particulares y específicas surge el último proceso de transmediación analizado, un regreso a la literatura de aquello que nació de la literatura fantástica y de la ciencia ficción: el surgimiento del LitRPG como género literario. Este género trae aparejado la irrupción de un nuevo verosímil fantástico, con lógicas de representación muy diferentes, por ejemplo, a las de Howard, Moorcock o el propio Tolkien, así como también introduce un nuevo vocabulario ajeno y extraño a la literatura, con términos y convenciones en extremo económicas pero altamente significativas que obedecen a estas nuevas lógicas de representación y comunicación. 


\section{Notas}

1. Queremos destacar que los ejemplos siguientes han sido rescatados de nuestro acervo personal de experiencias, y que, aunque por supuesto los consideramos pertinentes y válidos, de ningún modo pretenden ser más que un comentario indicativo entre muchos otros igualmente relevantes. Por otro lado, también han sido elegidos por su relación al tema de nuestra discusión.

2. Bracken, A. (2015), Star Wars: A New Hope. The Princess, the Scoundrel, and the Farm Boy, Disney Lucasfilm Press; Gidwitz, A. (2015), Star Wars: The Empire Strikes Back. So You Want to Be a Jedi, Disney Lucasfilm Press; Angleberger, T. (2015), Star Wars: Return of the Jedi. Beware the Power of the Dark Side, Disney Lucasfilm Press; Blish, J. (2015), Star Trek: The Classic Episodes, Barnes \& Noble.

3. Vallese, R. \& Vallese, V. (1999), Planescape: Torment, Wizard of the Coast; Hess, R. (1999), Planescape: Torment. Original text by Chris Avellone and Colin McComb Novelization by Rhyss Hess, Interplay Productions.

4. Para un excelente panorama de esta cuestión, cf. Peterson, J. (2012), Playing at the World: A History of Simulating Wars, People and Fantastic Adventures, from Chess to RolePlaying Games, San Diego, Unreason Press. Para el tema mencionado cf. pp. 204-250.

5. Tresca, M. (2011), pp. 5-6, esp.: "The Hobbit and The Lord of the Rings helped shape fantasy games through Tolkien's obsessive attention to detail and world building. Unlike typical 'sword and sorcery' novels, Tolkien's works spanned epic struggles, incorporating realworld languages and mythologies. This is not to lessen the contributions of other authors to fantasy gaming-Robert E. Howard, Michael Moorcock, Fritz Leiber, and H.P. Lovecraft are all major contributors"

6. Para un panorama de la historia y las problemáticas respecto a este tema, cf. Barton, $\mathrm{M}$ (2008).

7. Todas las traducciones son nuestras.

8. Resulta un lugar común en los CRPG que los primeros combates de los personajes sean contra enemigos (mobs) accesibles, para que los jugadores puedan familiarizarse con la interfaz y las mecánicas del juego. Yu Wo, 1/2 Prince: The Beginning of a Legend, p. 22: "Man-eating slimes, here I come! The first thing I saw were a dozen of man-eating slimes dotting the landscape. It's a good thing they won't agro, otherwise I'd have died of shock when they came charging over, I thought. Looking at the small knife in my hand, I realized that I seemed to have absolutely no experience whatsoever with melee combat." Cf. también el principio del clásico CRPG Fallout (1997).

\section{Referencias bibliográficas:}

Akella, G. (2016). Patch 17 (Realm of Arkon, Book 1), versión e-book, recuperado de Amazon.com services LLC.

Barton, M. (2008). Dungeons and Desktops. The History of Computer Role-Playing Games. Natick: A. K. Peters Ltd. 
Barton, M. (2007). Fun with PLATO, recuperado de armchairarcade.com website: http:// www.armchairarcade.com/neo/node/1396.

Campbell, J. (2008). The Hero with a Thousand Faces. Novato: New World Library.

Clute, J. y Grant, J. (1997). The Encyclopedia of Fantasy. New York: St. Martin's Griffin.

Howard, R. (2015). Conan: The Barbarian complete collection, versión e-book, recuperado de Amazon.com services LLC.

Gygax, G. (1979). Dungeon Master Guide. Advanced Dungeons and Dragons. Lake Geneva: TSR Games.

Konstik, C. (2018). What is LitRPG? Recuperado de https://www.levelup.pub/what-is-litrpg/ Konzack, L. (2018). Transmediality. M. Wolf (ed.) The Routledge Companion to Imaginary Worlds (pp. 134-140). New York and London: Routledge.

Mahanenko, V. (2015). Survival quest (Way of the Shaman: Book 1), versión e-book, recuperado de Amazon.com services LLC.

Parshall, J. y Tully, A. (2007). Shattered Sword. The Untold Story of the Battle of Midway. Dulles: Potomac Books Inc.

Peterson, J. (2012). Playing at the World: A History of Simulating Wars, People and Fantastic Adventures, from Chess to Role-Playing Games. San Diego: Unreason Press.

Rus, D. (2014). AlterWorld. Play to Live (Book 1), versión e-book, recuperado de Amazon. com services LLC.

Suvin, D. (1979) Metamorphoses of Science Fiction. On the Poetics and History of a Literary Genre. New Haven: Yale University Press.

Tolkien, J. (1975). Tree and Leaf, Smith of Wooton Major, The Homecoming of Beorhtnoth. Boston: Unwin Paperbacks.

Tolkien, J. (1991). The Lord of the Rings. Part One: The Fellowship of the Ring. London: Harper Collins Publishers.

Todorov, T. (2006). Introducción a la literatura fantástica. Buenos Aires: Ed. Paidós

Tresca, M. (2011). The Evolution of Fantasy Role Playing Games. Jefferson: MacFarland \& Co. William, T. (1998). Otherland: City of Golden Shadow, versión e-book, recuperado de Amazon.com services LLC.

Yu Wo (2004). 1/2 Prince: The Beginning of a Legend. Recuperado de https://www.princerevolution.org/halfprince-main/hp-online-viewer/

\begin{abstract}
In this paper we analyze a transmedial journey that ends on the creation of a new genre, the LitRPG (Literary RPG). This process begins in the seventies when certain literatures and prominent authors, like Tolkien, Lovecraft, Moorcock, Howard et al., are interactivized with the creation of the first RPG (Role Playing Games). Later, with the appearance of the new cybernetic platforms, these games are transmediated to give birth to the CRPG (Computer RPG), MUD (Multi User Domain/ Dungeon) and finally the MMORPG (Massive Multiplayer On Line RPG), whose logics and discursive characteristics appears abruptly in the literary field through the LitRPG novels, generating a return to literature while being deinteractivated.
\end{abstract}


Apart from the analysis of the transmedia process, we analyze some of the main characteristics of this genre by reading four authors deemed the pioneers of the genre - D. Rus, G. Akella, V. Mahanenko y Yu Wo. We will also look upon how these characteristics are present in certain precursors, (in the sense of Borges' "Kafka y sus precursors").

Keywords: LitRPG - D. Rus - G. Akella - V. Mahanenko - Yu Wo - Transmediality - RPG - MMORPG

Resumo: Neste artigo percorremos um roteiro transmidiático que tem como resultado o surgimento de um novo gênero literário, o LitRPG (Literary RPG). Esse proceso começa na década de 70 quando se interativisam certas literaturas e autores eminentes como Tolkien, Lovecraft, Moorcock, Howard et al. e aparecem os primeiros RPG ( Role Playing Games), logo, esses são transmediados aos novos suportes cibernéticos para dar origem aos CRPG ( computer RPG), MUD ( Multi user Domain/ Dugeon) e finalmente os MMORPG (Massive Multiplayer Online RPG), cujas lógicas e características discursivas se apresentam no terreno literário através das obras de litRPG e geram um retorno à literatura a partir de um processo de desenterativisazação.

Além de analisar o processo de transmidiação, analisam-se também as características principais desse novo gênero, nos adentrando na obra de quatro autores considerados pioneiros - D. Rus, G. Akella, V. Mahanenko y Yu Wo - assim como também em certas características pré-figuradas em certos precursores do gênero (em termos de "Kafka e seus precursores" de J. L. Borges).

Palavras chave: LitRPG - D. Rus - G. Akella - V. Mahanenko - Yu Wo - Transmediación - RPG - MMORPG

[Las traducciones de los abstracts fueron supervisadas por el autor de cada artículo] 